\title{
Dental consultations in UK general practice and antibiotic prescribing rates:
}

\author{
a retrospective cohort study
}

\begin{abstract}
Background

The frequency of consulting for dental problems in general medical practice, and antibiotic prescribing associated with these consultations, is poorly described.

\section{Aim}

To describe consultation rates and antibiotic use for dental problems in UK general medical practice, and explore factors associated with antibiotic prescribing for dental conditions.

\section{Design and setting}

A retrospective cohort study using Clinical Practice Research Datalink, a database of general practice patient records in the UK

\section{Method}

All dental consultations between 2004 and 2013 were identified. The main outcome was the prescription of an antibiotic during a dental consultation. Multilevel logistic regression was conducted to examine factors associated with antibiotic prescription.
\end{abstract}

\section{Results}

In all, 288169 dental consultations were included in the cohort. The average rate of dental consultations was 6.06 consultations per 1000 patient-years. Rates of dental consultation decreased from 6.84 consultations per 1000 patient-years in 2008, to 4.23 consultations per 1000 patient-years in 2013. Consultation rates were higher among females than males and highest in patients aged $20-29$ years. An antibiotic was prescribed in $57.1 \%$ of consultations. Significant predictors $(P<0.001)$ of antibiotic prescribing included: patient middle age, male sex, and previous consultations for tooth-related problems. Antibiotics were more likely to be prescribed during consultations in December lodds ratio [OR] 1.18, 95\% confidence interval $[\mathrm{CI}]=1.13$ to $1.24, P<0.001$, reference month: June) and on a Monday (OR $1.10,95 \% \mathrm{Cl}=1.07$ to 1.13, $P<0.001$ ) or a Friday (OR $1.15,95 \% \mathrm{Cl}=1.12$ to $1.18, P<0.001$, reference day: Wednesdayl.

\section{Conclusion}

Consultation rates for dental problems in UK general practice are relatively low but more than half result in the prescription of an antibiotic. This raises concerns about patient morbidity and contributions to antimicrobial resistance.

\section{Keywords}

antibacterial agents; dental care; dentistry; general practice; inappropriate prescribing: physician's practice patterns.

\section{INTRODUCTION}

When experiencing a dental problem, some patients may consult a GP instead of a dentist. ' This may be due to difficulties accessing dental services, patient perceptions of medical and dental practitioners' scope of practice, referred or poorly differentiated pain, perceived need for antibiotics, or financial concerns about the cost of dental treatment. ${ }^{2,3}$

Most dental problems cannot be comprehensively managed in general practice. Clinical guidelines recommend that the first-line treatment for acute dental conditions should be an operative intervention, such as extraction or endodontic (root canal) treatment, 4,5 yet GPs lack the specialist knowledge, skills, and facilities necessary to undertake such procedures. Instead, patients who visit their GP with tooth-related problems are more likely to be prescribed a systemic antibiotic than those who consult a dentist. ${ }^{6}$ However, antibiotics are of little clinical benefit for many acute dental conditions, ${ }^{7.8}$ and their indiscriminate use may increase the risk of both adverse reactions and the emergence of antimicrobial resistant bacterial strains. Furthermore, patients who do not receive operative treatment for acute dental problems may be at greater risk of developing a severe infection. ${ }^{9}$

Despite concerns within the general medical profession that attendance for

AL Cope, PhD, BDS, specialty trainee in dental public health, Cardiff and Vale University Health Board, University Dental Hospital, Cardiff. IG Chestnutt, BDS, MPH, PhD, FDS(DPH)RCSEd, professor and honorary consultant in dental public health, School of Dentistry; F Wood, PhD, senior lecturer; NA Francis, PhD, MD, BA, PGD (Epidemiology), MRCGP, clinical reader, Division of Population Medicine, School of Medicine, Cardiff University, Cardiff. dental problems is increasing, ${ }^{10,11}$ there has been little attempt to quantify the burden of dental consultations and associated antibiotic use within UK general practice in the last decade. Although a previous study reported that the rate of dental consultations in general practice in Wales in 1996 was 6.90 per 1000 patient years, there have been significant changes in provision of NHS dentistry in England and Wales since then, which may have modified access to dental services in these areas. As access to dental services is likely to be a key driver of dental consultations in general practice, rates of consultations may have changed from those reported previously. ${ }^{1}$ The objectives of the current study were therefore to describe rates of attendance for dental problems in UK general practice, to characterise the use of antibiotics in such consultations, and to describe patient, practice, and consultation characteristics predictive of antibiotic prescription.

\section{METHOD}

\section{Design}

Retrospective cohort study of dental consultations in UK general practice using routinely collected data from primary care records held within the Clinical Practice Research Datalink (CPRD).

\section{Study population}

Dental consultations were identified by

\section{Address for correspondence}

Anwen L Cope, Room 108, University Dental Hospital, Heath Park, Cardiff CF14 4XY.

E-mail: copea1Acardiff.ac.uk

Submitted: 8 Oct 2015; Editor's response: 2 Nov 2015; final acceptance: 30 Nov 2015.

\section{(C)British Journal of General Practice}

This is the full-length article (published online 30 Mar 2016) of an abridged version published in print. Cite this article as: Br J Gen Pract 2016; DOI: 10.3399/bjgp16X684757 


\section{How this fits in}

Despite GP's concerns about demands on their services, there has been little attempt to quantify the number of dental consultations, or antibiotic prescribing for dental problems, in UK general practice in recent years. This study demonstrates that consultation rates for dental problems, although generally low, vary between settings and may place a substantial burden on some practices. Overall, attendances for dental problems decreased between 2008 and 2013. More than half of patients who consult their GP with a dental problem are prescribed an antibiotic, despite evidence that it is unlikely to confer clinical benefit for most acute dental conditions. Antibiotic prescribing for dental conditions is more common in patients who are middle-aged, male, have had a previous consultation for a tooth-related problem, and in those who consult on Mondays and Fridays, and in December.

one of 103 diagnostic or examination Read Codes related to pathologies of the teeth or periodontium (further details available from the authors). Read Codes related to nondental pathologies of the oral cavity, such as salivary gland disease, were excluded.

All dental consultations with an event date between 1 January 2004 and 31 December 2013 (inclusive) were extracted for analysis. The data were then cleaned according to CPRD-approved protocols (Figure 1).

\section{Primary outcome}

The primary outcome of the study was whether a systemic antibiotic was prescribed during a dental consultation. Antibiotic prescriptions were identified using drug codes within CPRD that map to section 5.1 of the British National Formulary. Drugs used to treat tuberculosis and leprosy were excluded as in a similar study. ${ }^{12}$

\section{Covariates}

The following patient, practice, and consultation-related characteristics were also recorded:

- patient age and sex;

- episode number;

- dentally-relevant comorbidities diabetes mellitus (type 1 and 2), rheumatoid arthritis, and coronary heart disease;

- day, month, and year of consultation;

- country of consultation; and

\section{- practice size.}

Patient age at consultation was calculated from month and year of birth, and the event date of the dental consultation. The age at last birthday was used in all analyses. Patient sex was directly extractable from the dataset.

Each episode within the dataset was numbered. An episode was considered to encompass all consultations made by a single patient for a discrete dental problem. An episode was defined as all dental consultations made by one patient with $\leq 30$ days separation between sequential consultations. Some patients consulted for more than one episode of dental problems during the study period (Figure 1).

Diabetes mellitus (type 1 and type 2),
Figure 1. Data flow diagram for selection of dental consultations in Clinical Practice Research Datalink (CPRD).

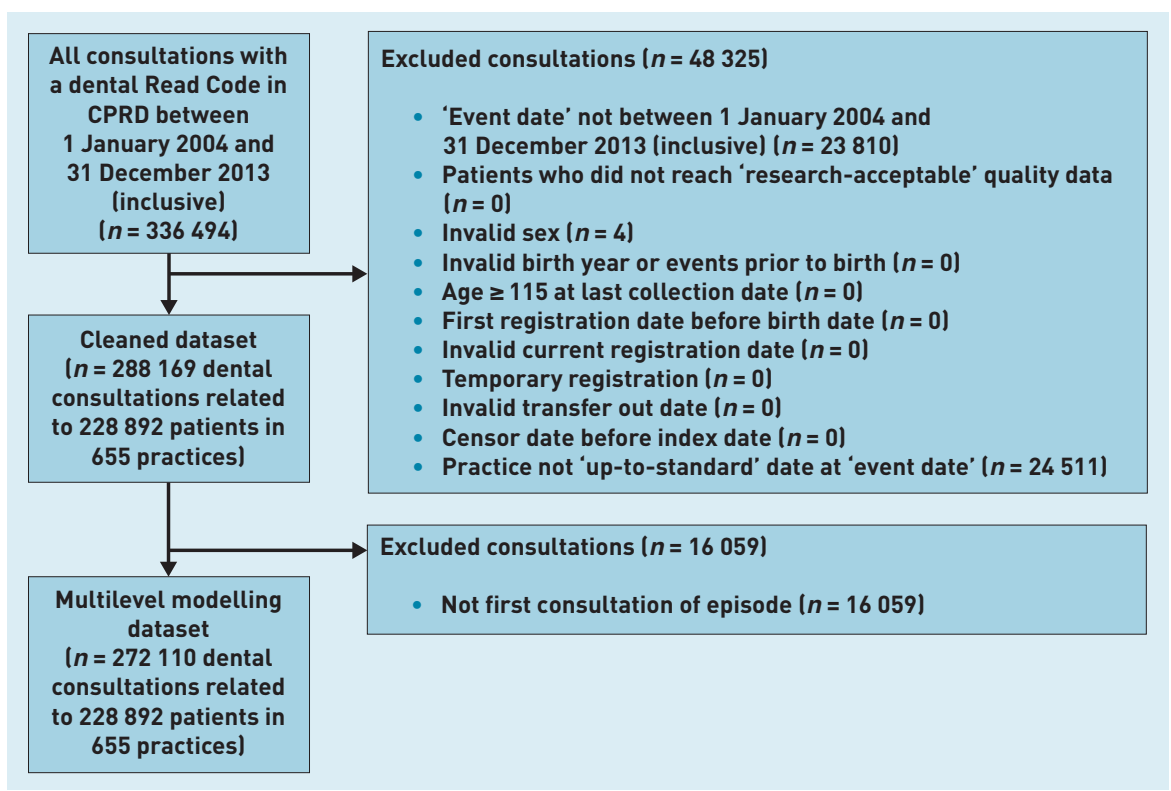


rheumatoid arthritis, and coronary heart disease have all been associated with development of dental disease. 13,14 Comorbid conditions were identified using Read Codes identified by previously published studies. ${ }^{15-17}$ A comorbidity was considered to be present if a patient had been recorded as having a consultation with a disease-specific Read Code on, or before, the event date of the dental consultation.

Day, month, and year of consultation, and country of consultation were directly extractable from the dataset. Because Saturday and Sunday appointments together made up less than $4 \%$ of all consultations, these were aggregated together to form the 'weekend' dummy variable for entry into the model.

Practice size was based on the average number of registered patients between 2004 and 2013. The quartiles were: $\leq 5508$ registered patients; 5509-7776 patients; 7777-10 830 patients, and $\geq 10831$ patients.

\section{Data analysis}

The annual rate of dental consultations per 1000 patient-years and proportion of dental consultations resulting in an antibiotic prescription were calculated, together with rates by age and sex. Consultation rates were also calculated for each practice. All rates were calculated using CPRD denominator data. Data were analysed using IBM SPSS (version 20.0).

To identify factors associated with antibiotic prescribing, a multilevel logistic regression was performed. This approach accounted for the hierarchical structure of CPRD data, with consultations clustered within patients, who are clustered within general practices. The binary response prescription for dental problems in UK general medical practice 2004-2013. variable was whether an antibiotic was prescribed and only the first consultation within an episode was entered into the model. Patient, practice, and consultationrelated explanatory variables were selected on the basis of existing literature, or where a convincing argument could be made for their inclusion. All explanatory variables were screened for collinearity. The three-level random intercepts model was converged using an iterative generalised least squares algorithm. The first order marginal quasi likelihood (MQL) approximation was fitted first, followed by the second order penalised quasi-likelihood (PQL). ${ }^{18}$ Modelling was performed using MLwiN, (version 2.28). The statistical significance of individual parameters in the model was calculated using the Wald test. The level of significance was set at 0.05 .

\section{RESULTS}

Following data cleaning there were 288169 dental consultations with an event date between 1 January 2004 and 31 December 2013 (inclusive) (Figure 1). These related to 228892 patients who consulted at one of 655 CPRD-contributing general practices. Most of the practices were in England (507 out of $655,77.4 \%$ ), followed by Scotland (76 out of $655,11.6 \%$ ), Wales (50 out of 655 , $7.6 \%$ ), and Northern Ireland (22 out of 655 , $3.4 \%)$.

\section{Consultation rates}

The average rate of dental consultations was 6.06 consultations per 1000 patient-years (95\% confidence interval $[\mathrm{Cl}]=6.03$ to 6.08 ). Rates of consultations increased slightly between 2004 and 2008 before declining between 2008 and 2013 (Figure 2). Rates of dental consultations varied between practices, ranging from 0.06 to 29.8 dental consultations per 1000 patient-years (Figure 3). Rates of dental consultation were higher among females than males and in patients aged 20-29 years (Figure 4).

\section{Antibiotic prescribing}

During the study period there were 3.46 antibiotic prescriptions for dental problems per 1000 patient-years (Figure 2). Just over half (164 432 out of $288169,57.1 \%)$ of dental consultations resulted in the prescription of an antibiotic.

In total, 180651 antibiotic items were prescribed, in 16219 consultations lout of 164 432, 9.9\%) more than one antibiotic was prescribed. Penicillins such as amoxicillin were the most commonly prescribed antibiotics (12654 out of $180651,69.8 \%$ ), followed by metronidazole and tinidazole 


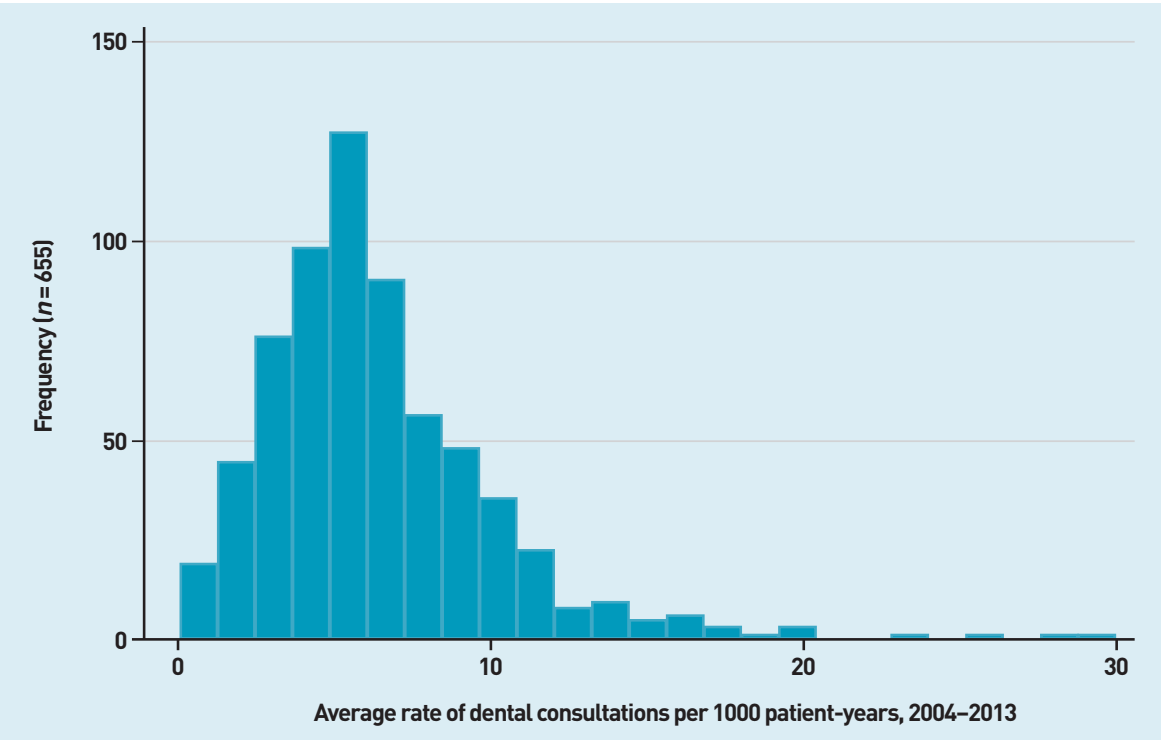

Figure 3. Distribution of average rate of dental consultations per 1000 patient-years for 655 Clinical Practice Research Datalink (CPRD)-contributing UK general medical practices 2004-2013.

Figure 4. Rates of dental consultation in UK general medical practice, by age and sex of patient 2004-2013.
(32 774 out of $180651,18.1 \%$ ), macrolides (15 655 out of $180651,8.7 \%$ ), and cephalosporins (3545 out of $180651,2.0 \%$ ). Tetracyclines and clindamycin were also prescribed on a small number of occasions.

\section{Predictors of antibiotic prescribing}

Table 1 presents the characteristics of the 272110 dental consultations used within the multilevel modelling. Figure 5 shows the results of the multilevel logistic regression, indicating predictors of antibiotic prescription during a dental consultation in UK general practice.

Patients of middle age (40-59 years) were more likely to receive an antibiotic than older or younger patients $(P<0.001)$, The association between age and antibiotic prescribing follows a quadratic distribution: the odds ratios (ORs) associated with this

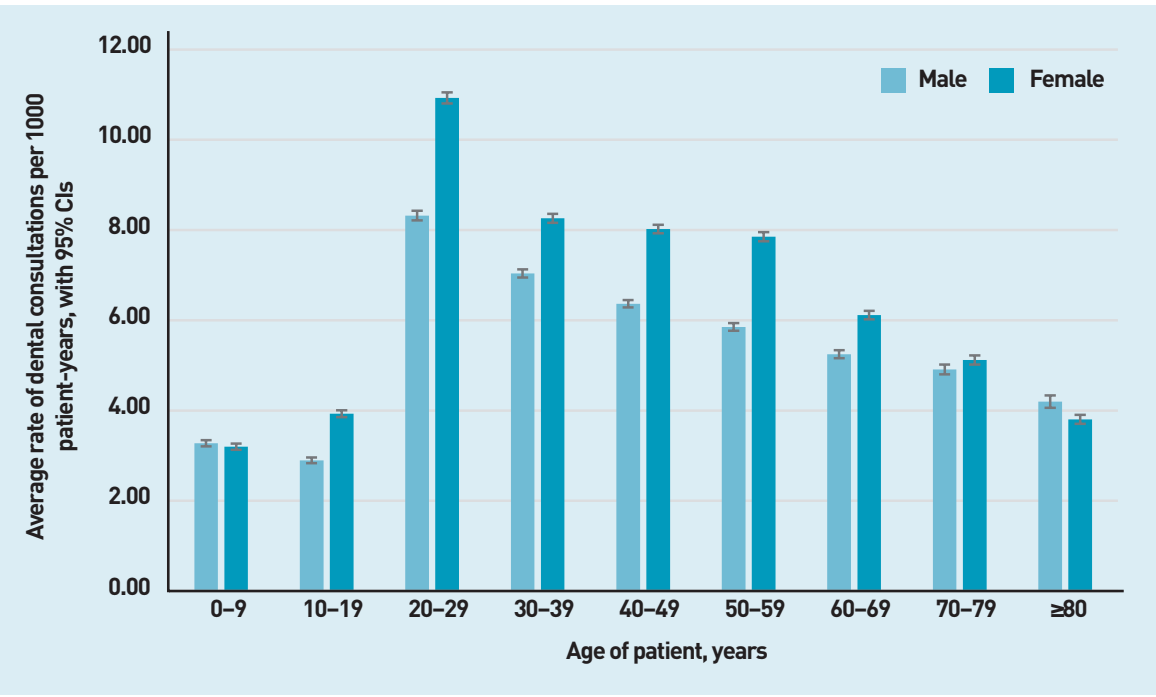

\section{Table 1. Characteristics of the dataset of dental consultations used in multilevel modelling}

\begin{tabular}{|c|c|}
\hline & $\begin{array}{l}\text { Consultations } \\
(n=272110)\end{array}$ \\
\hline Antibiotic prescribed, $n(\%)$ & $156243 a(57.4)$ \\
\hline \multicolumn{2}{|c|}{ Patient demographic characteristics } \\
\hline $\begin{array}{l}\text { Mean age at consultation, years } \\
\text { (SD) }\end{array}$ & $41.3(19.9)$ \\
\hline Female, $n(\%)$ & $149470(54.9)$ \\
\hline $\begin{array}{l}\text { Episodes of dental problems per } \\
\text { patient, median (IQR) }\end{array}$ & $1.0(1.0-1.0)$ \\
\hline \multicolumn{2}{|l|}{ Patient comorbidities, $n(\%)$} \\
\hline Diabetes mellitus (type 1 or 2 ) & $12387(4.6)$ \\
\hline Rheumatoid arthritis & $2003(0.7)$ \\
\hline Coronary heart disease & $12017(4.4)$ \\
\hline \multicolumn{2}{|l|}{ Day of consultation, $n(\%)$} \\
\hline Monday & $60443(22.2)$ \\
\hline Tuesday & $51691(19.0)$ \\
\hline Wednesday (base) & $47853(17.6)$ \\
\hline Thursday & $47799(17.6)$ \\
\hline Friday & $54662(20.1)$ \\
\hline Weekend (Saturday or Sunday) & 9662 (3.5) \\
\hline \multicolumn{2}{|l|}{ Month of consultation, $n(\%)$} \\
\hline January & $23386(8.6)$ \\
\hline February & $21386(7.9)$ \\
\hline March & $23340(8.6)$ \\
\hline April & $21796(8.0)$ \\
\hline May & $22358(8.2)$ \\
\hline June (base) & $22957(8.4)$ \\
\hline July & 23159 (8.5) \\
\hline August & 22614 (8.3) \\
\hline September & $22955(8.4)$ \\
\hline October & $22835(8.4)$ \\
\hline November & 22407 (8.2) \\
\hline December & $22917(8.4)$ \\
\hline \multicolumn{2}{|l|}{ Country, $n(\%)$} \\
\hline England (base) & 221973 (81.6) \\
\hline Scotland & $19612(7.2)$ \\
\hline Wales & 25179 (9.3) \\
\hline Northern Ireland & $5346(2.0)$ \\
\hline \multicolumn{2}{|l|}{ Practice size, $n(\%)$} \\
\hline $\begin{array}{l}1^{\text {st }} \text { quartile ( } \leq 5508 \text { registered } \\
\text { patients) (base) }\end{array}$ & $31660(11.6)$ \\
\hline $2^{\text {nd }}$ quartile (5509-7776 patients) & 53925 (19.8) \\
\hline $3^{\text {rd }}$ quartile (7777-10 830 patients) & $73763(27.1)$ \\
\hline $4^{\text {th }}$ quartile ( $\geq 10831$ patients) & $112762(41.4)$ \\
\hline
\end{tabular}

are $1.59(95 \% \mathrm{Cl}=1.57$ to 1.62$)$ for age at consultation (per 10-year increase) and $0.95(95 \% \mathrm{Cl}=0.95$ to 0.95$)$ for age at consultation (per 10-year increase). ${ }^{2}$ Male patients were marginally more likely to receive an antibiotic than females IOR 1.08, $95 \% \mathrm{Cl}=1.06$ to $1.09, P<0.001)$. Patients who had previously consulted their GP 


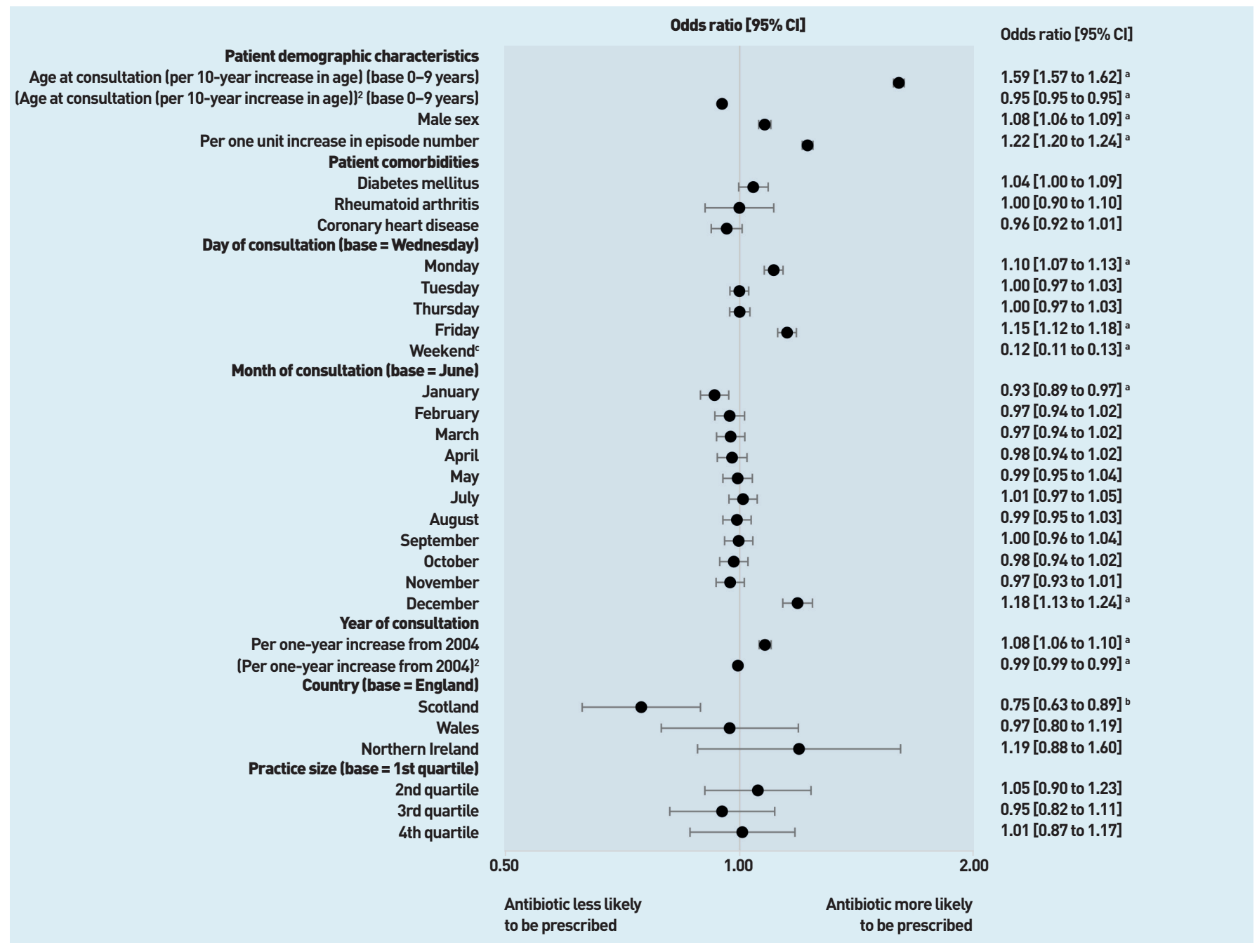

Figure 5. Three-level, random intercepts logit model for predictors of antibiotic prescribing in a dental consultation in UK general practice. ${ }^{a} P<0.001 .{ }^{b} P<0.01$. cnot shown. with a dental problem within the study period were more likely to be prescribed an antibiotic in each subsequent episode (OR 1.22, $95 \% \mathrm{Cl}=1.20$ to $1.24, P<0.001$ ). None of the selected comorbidities were significantly associated with differences in antibiotic prescribing for dental problems once other variables were controlled for.

Consultations for dental problems that occurred on a Monday (OR 1.10, $95 \% \mathrm{Cl}=1.07$ to $1.13, P<0.001$ ) or a Friday (OR 1.15, 95\% Cl=1.12 to $1.18, P<0.001$ ) were significantly more likely to result in an antibiotic prescription than those on a Wednesday (the reference day). There were no significant differences between Wednesday consultations and those on either a Tuesday or Thursday. Consultations for dental problems that had a weekend event date were significantly less likely to be associated with an antibiotic prescription than on the reference day IOR 0.12, $95 \% \mathrm{Cl}=0.11$ to $0.13, P<0.001)$.

When month of consultation was considered, the likelihood of antibiotic prescribing was higher in December (OR 1.18, 95\% Cl=1.13 to 1.24, $P<0.001$ ) and lower in January (OR 0.93, 95\% Cl = 0.89 to $0.97, P<0.001$ ) compared to June (the reference month). There were no other significant differences between months.

Dental consultations that occurred in Scotland were significantly less likely to result in an antibiotic than those in England (OR 0.75, 95\% Cl=0.63 to 0.89, P<0.01). There were no significant differences between patients in England and either Wales or Northern Ireland. Practice size was not a predictor of antibiotic prescription.

\section{DISCUSSION}

\section{Summary}

This is the first study in the past decade to comprehensively quantify the burden of dental consultations on UK general practice. During the study period a typical general practice with 7000 registered patients would have expected to see between 30 and 48 patients with dental 
problems per year. However, there was substantial inter-practice variation, and this figure may be as high as 209 dental consultations per year for a 7000-patient practice. Despite clinical guidance recommending that antibiotics should not be prescribed for dental conditions in the absence of adjunctive dental treatment, 4,5 an antibiotic was given in more than half of consultations. Middle-aged, male patients who had previously consulted their GP with tooth-related problems were most likely to receive an antibiotic. Antibiotics were more likely to be prescribed in consultations on a Monday or a Friday than those midweek, and patients consulting in December were more likely to be prescribed an antibiotic than at other times of the year. Consultations that occurred in Scotland were less likely to result in an antibiotic prescription than those in England.

\section{Strengths and limitations}

A principal strength of this study is the large, representative sample of UK general practice consultations included in the analyses. This gave sufficient statistical power to examine patient, practice, and consultation characteristics predictive of antibiotic prescription, and increases the external validity of the findings. However, data held within CPRD are primarily collected for clinical practice rather than for research, and therefore limitations exist with regard to their use. Although applying the CPRD's data quality metrics facilitated the removal of records in which there were obvious data quality problems, there may still be coding imperfections and variations in coding habits between practices. Additionally, the manner in which consultations are coded in CPRD means that it is difficult to separate different elements within an individual clinical encounter, particularly when patients consult with a 'shopping list' of problems. In a small number of instances this may have led to the incorrect labelling of antibiotic prescribing within a dental consultation.

The current study was able to identify a number of factors relating to the patient, practice, or consultation associated with antibiotic prescribing. However, no reliable measures of dental infection severity or socioeconomic deprivation were available from CPRD. Furthermore, within the multilevel modelling process, consultations were assigned an episode number based on their number of previous consultations within the dataset. This variable is subject to a non-differential misclassification bias as the initial consultation does not necessarily coincide with the first-ever consultation with a GP for a dental problem. For example, a patient may have consulted pre-2004 or at a non-CPRD registered practice, potentially resulting in biased estimates of the coefficients. A longer study period or a dynamic regression model, in which previous responses can exert a causal influence on subsequent responses, may have provided a more accurate representation of how previous episodes affect the outcome of current consultations. ${ }^{19}$

\section{Comparison with existing literature}

The findings of this study are largely consistent with another study which reported that, although rates of attendance for dental problems in general practice are relatively low, they can vary substantially between practices. ${ }^{1}$

Consultations for dental problems, while less frequent than for other common infections ${ }^{20}$ may still place a substantial burden on some general practices. Despite the concerns of GPs that rates of dental consultations are increasing, ${ }^{10,11}$ the current study demonstrates the contrary; that among the CPRD-contributing practices, rates of dental consultations fell between 2008 and 2013. It is unclear whether this may be due to improved availability of dental services, changes in access to general practices, refusal of GPs to treat dental conditions, or more patients seeking care from accident and emergency departments.

Consultation rates for dental problems were higher for females than males. This is consistent with the findings of other studies for both dental problems, ${ }^{1}$ and other conditions. ${ }^{21}$ The highest rates of dental consultation at general practices was found among patients aged 20-29 years. Not only do young adults have a higher prevalence of lower third molar lwisdom tooth) pathologies and dental caries than the general population, ${ }^{22}$ they are also the least likely group of patients to have an established general dental practitioner or to engage in routine dental visits. ${ }^{23}$

Within the population studied, over half of consultations for dental problems resulted in the prescription of an antibiotic. While fewer patients attending their GP with dental problems may be receiving antibiotics than previously reported, ${ }^{6}$ the widespread use of antibiotics in the management of toothrelated complaints in general practice is still alarming.

Antibiotics are unlikely to result in longterm resolution of dental conditions, yet carry with them risks of adverse reaction 
and increase the selective pressures leading to the development of antibiotic resistance. In addition, evidence also suggests that prescribing antibiotics for infections may increase medical consultation rates for the same condition in the future. ${ }^{24}$ Therefore, there is concern that receiving a treatment, such as an antibiotic, from a GP for a dental problem may incorrectly reinforce patients beliefs that general practice is an appropriate place to consult when experiencing dental problems, ${ }^{3}$ fuelling future consultations for tooth-related problems.

The finding that patients consulting on a Monday or Friday were more likely to receive an antibiotic is in keeping with findings relating to all-cause antibiotic prescribing in general medical practice. ${ }^{25}$ Similarly, patients consulting in December were also more likely to be prescribed antibiotics than those in the reference month (June). This may be a result of GPs' anxieties regarding patients' access to regular dental care providers over the Christmas period.

\section{Implications for research and practice}

Large population-based studies of dental consultations in general practice are scarce. This work therefore provides new insight into the burden of dental consultations in UK primary care. Although findings indicate that dental consultations may not place undue strain on the resources of most general practices, high rates of antibiotic prescribing in dental consultations raise concerns about possible patient morbidity from untreated dental disease and potential impact on rates of antimicrobial resistance. GPs should avoid prescribing antibiotics where possible, and further work is required to identify the reasons for prescribing and also how patients experiencing dental problems could be best directed to emergency dental services. Additional research is also needed to identify the reasons for the apparent decrease in consultation rates for dental problems in general practice as well as the substantial variation in consultation rates for dental problems between practices. Understanding the drivers of antibiotic prescribing for dental problems will inform educational interventions for GPs to ensure patients receive the most appropriate care for their dental problems.

\section{Funding}

Anwen $L$ Cope was supported by a Cardiff University Presidents' Research Scholarship, reference RCUC099.

\section{Ethical approval}

Ethical approval was granted by the CPRD Independent Scientific Advisory Committee (ISAC), reference 14_144R.

\section{Provenance}

Freely submitted; externally peer reviewed.

\section{Competing interests}

The authors have declared no competing interests.

\section{Acknowledgements}

The authors acknowledge the contributions of Hywel Jones (Cardiff University) and the Centre for Improving Population Health through E-Health Research (CIPHER) for extracting the data from CPRD. Also, Chris Poole (Cardiff University) for his advice on an early version of the study protocol, and Sara Jenkins-Jones from Pharmatelligence (www.pharmatelligence.co.uk) for her assistance in extracting data from CPRD during the development of the study.

\section{Discuss this article}

Contribute and read comments about this article: bjgp.org/letters 


\section{REFERENCES}

1. Anderson R, Richmond S, Thomas DW. Patient presentation at medical practices with dental problems: an analysis of the 1996 General Practice Morbidity Database for Wales. Br Dent J 1999: 186: 297-300.

2. Bell GW, Smith GLF, Rodgers JM, et al. Patient choice of primary care practitioner for orofacial symptoms. Br Dent J 2008; 204: 669-673.

3. Cope AL, Wood F, Francis NA, Chestnutt IG. General practitioners' attitudes towards the management of dental conditions and use of antibiotics in these consultations: a qualitative study. BMJ Open 2015; 5: e008551.

4. Scottish Dental Clinical Effectiveness Programme. Drug prescribing for dentistry: dental clinical guidance. 2nd ed, 2011. http://www.sdcep.org.uk/ wp-content/uploads/2013/03/Drug_Prescribing for Dentistry 2 Web.pdf (accessed 6 Mar 2016)

5. Palmer NOA, Longman L, Randall C, Pankhurst CL. Antimicrobial prescribing for general dental practitioners. 2nd edn. London: Faculty of General Dental Practitioners (UK), 2012.

6. Anderson R, Calder L, Thomas DW. Antibiotic prescribing for dental conditions: general practitioners and dentists compared. Br Dent J 2000; 188: 398-400.

7. Fedorowicz Z, van Zuuren EJ, Farman AG, et al. Antibiotic use for irreversible pulpitis. Cochrane Database Syst Rev2013; 12: CD004969.

8. Cope A, Francis N, Wood F, et al. Systemic antibiotics for symptomatic apical periodontitis and acute apical abscess in adults. Cochrane Database Syst Rev 2014; 6: CD010136

9. Seppanen L, Lemberg KK, Lauhio A, et al. Is dental treatment of an infected tooth a risk factor for locally invasive spread of infection? J Oral Maxillofac Surg 2011; 69: 986-993.

10. Bint A. NHS dentistry: General practitioners are doing dentists' work. BMJ 2008; 336: 1088

11. Matthews-King A. Nine out of $10 \mathrm{GPs}$ feel 'under pressure' from patients with dental complaints. Pulse 2013; 18 Nov: http://www.pulsetoday.co.uk/news/ clinical-news/nine-out-of-ten-gps-feel-under-pressure-from-patients-withdental-complaints/20005094.article\#.VEo8t_LrYYg laccessed 16 Mar 2016).

12. Gulliford MC, Dregan A, Moore MV, et al. Continued high rates of antibiotic prescribing to adults with respiratory tract infection: survey of $568 \mathrm{UK}$ general practices. BMJ Open 2014; 4: e006245.

13. Cullinan MP, Ford PJ, Seymour GJ. Periodontal disease and systemic health: current status. Aust Dent J 2009; 54 Suppl 1: S62-69.

14. Koziel J, Mydel P, Potempa J. The link between periodontal disease and rheumatoid arthritis: an updated review. Curr Rheumatol Rep 2014; 16: 408.

15. Gray J, Orr D, Majeed A. Use of Read Codes in diabetes management in a south London primary care group: implications for establishing disease registers. BMJ 2003; 326: 1130 .

16. Nicholson A, Ford E, Davies KA, et al. Optimising use of electronic health records to describe the presentation of rheumatoid arthritis in primary care: a strategy for developing code lists. PLoS One 2013; 8: e54878.

17. Bhattarai N, Charlton J, Rudisill C, Gulliford MC. Coding, recording and incidence of different forms of coronary heart disease in primary care. PLoS One 2012; 7: e29776.

18. Leckie G, Charlton C. runmlwin - A program to run the MLwiN multilevel modelling software from within Stata. J Statistical Software 2013; 52: 1-40.

19. Steele F. Multilevel modelling of repeated measures data. 2014. Stata Practical. LEMMA VLE Module 15, 1-61. http://www.bristol.ac.uk/cmm/learning/course. html ; actual URL: http://mww.bristol.ac.uk/media-library/sites/cmm/migrated/ documents/15-stata-example.pdf laccessed 6 Mar 2016).

20. Currie CJ, Berni E, Jenkins-Jones S, et al. Antibiotic treatment failure in four common infections in UK primary care 1991-2012: longitudinal analysis. BMJ 2014; 349: g5493.

21. Hippisley-Cox J, Vinogradova Y. Final report to the NHS Information Centre and Department of Health. Trends in consultation rates in general practice 1995/1996 to 2008/2009: Analysis of the QResearch $\otimes$ database. 2009. http:// unw.hscic.gov.uk/catalogue/PUB01077/tren-cons-rate-gene-prac-95-09-95-09rep.pdf (accessed 6 Mar 2016)

22. White D, Pitts N, Steele J, et al. 2: Disease and related disorders - a report from the Adult Dental Health Survey 2009. Leeds: The Health and Social Care Information Centre, 2011.

23. Morris J, Chenery V, Douglas G, Treasure E. 6: Service considerations - a report from the Adult Dental Health Survey 2009. Leeds: The Health and Social Care Information Centre, 2011.

24. Little P. Gould C. Williamson I, et al. Reattendance and complications in a randomised trial of prescribing strategies for sore throat: the medicalising effect of prescribing antibiotics. BMJ 1997; 315: 350-352.

25. Kuehlein T, Szecsenyi J, Gutscher A, Laux G. Antibiotic prescribing in genera practice - the rhythm of the week: a cross-sectional study. J Antimicrob Chemother 2010; 65: 2666-2668. 